\title{
Research and automation of the process of electrocoagulation wastewater treatment on the basis of the developed mathematical model
}

https://doi.org/10.31713/MCIT.2020.17

Andrii Safonyk

National University of Water and Environmental Engineering Institute of Automatics, Cybernetics and Computer

Engineering

Rivne, Ukraine

a.p.safonyk@nuwm.edu.ua

Olga Safonyk

Rivne Humanitarian Gymnasium, Rivne, Ukraine

Rivne, Ukraine

olya.safonyk@gmail.com

\author{
Sergiy Koval \\ National University of Water and Environmental Engineering \\ Institute of Automatics, Cybernetics and Computer \\ Engineering \\ Rivne, Ukraine \\ v.a.pasichnyk@nuwm.edu.ua
}

Andre Batako

Liverpool John Moores University,

Liverpool, UK

a.d.batako@ljmu.ac.uk
Abstract- The paper presents the principles of the construction of an automated wastewater treatment system based on a generalized spatial model. A model problem, which contains descriptions of the main processes occurring in the electrocoagulation reactor taking into account the placement of the plates was developed. To verify the adequacy, a comparison between computer simulations and the results of a field experiment was made. The influence of reactor size, current density, location of electrode plates on the efficiency of chromium extraction was investigated. As a result of the study, it was proved that the mathematical model is effective for determining the parameters of the purification process, and the obtained coefficients are effective for optimizing the purification process. Based on these studies, a scheme for automation of the process of wastewater treatment from chromium was built. With the help of SCADA - WinCC, a flexible control system with the ability to control the performance of the entire installation in real time was developed.

Keywords - Mathematical model of electrocoagulation process, singularly perturbed problem, asymptotic method, automated system.

\section{INTRODUCTION}

The quality of natural waters changes the most as a result of pollution by sewage from industrial enterprises and communal services, as well as from surface runoff from the territories of settlements, industrial sites, transport routes and agricultural lands.

Reagent method of purification and disposal of concentrated technological solutions and washing water is widely used as a method of sewage treatment in the production. Reagent methods include neutralization of acids and alkalis, transfer of ions to a slightly soluble state. One of the most progressive trends in water treatment technology is the development and implementation of electrochemical methods. Electrochemical methods (electrodialysis, electrochemical oxidation and hydrolysis) are methods related to the action of electric current on aqueous solutions.

\section{MATERIALS AND METHODS}

Let us consider the curvilinear parallelepiped (electrocoagulation unit) of $=A B C D A * B * C * D *$, limited by smooth orthogonal equipotential surfaces between themselves in angular points and ribs $A B B_{*} A_{*}=\left\{z: f_{1}(x, y, z)=0\right\}$, $C D D_{*} C_{*}=\left\{z: f_{2}(x, y, z)=0\right\}$ and also surfaces of flow $A D D_{*} A_{*}=\left\{z: f_{3}(x, y, z)=0\right\}, B C C_{*} B_{*}=\left\{z: f_{4}(x, y, z)=0\right\}$, $A B C D=\left\{z: f_{5}(x, y, z)=0\right\}, A_{*} B_{*} C_{*} D_{*}=\left\{z: f_{6}(x, y, z)=0\right\}$. In the paper, the mathematical model developed in [1-2] for a spatial case. Thus, to find the distribution of pollution concentration and temperature in the electrocoagulation unit, we come to the following model problem:

$$
\left\{\begin{array}{l}
\frac{\partial c}{\partial t}=-v_{c} \nabla c+\nabla\left(D_{c}(\bar{T}) \nabla c\right)-\alpha \bar{T} c-\Phi, \\
\frac{\partial \bar{T}}{\partial t}=v_{\bar{T}} \nabla \bar{T}+D_{\bar{T}} \Delta \bar{T}+\Psi,
\end{array}\right.
$$




$$
\begin{gathered}
\left.c\right|_{A B B_{*} A_{*}}=c_{*}(M, t),\left.\quad \frac{\partial c}{\partial \vec{n}}\right|_{C D D_{*} C_{*}}=0, \\
\left.\frac{\partial c}{\partial \vec{n}}\right|_{A D D_{*} A_{*} \cup B C C_{*} B_{*} \cup A B C D \cup A_{*} B_{*} C_{*} D_{*}}=0, \\
\left.\bar{T}\right|_{A B B_{*} A_{*}}=\bar{T}_{*}(M, t),\left.\quad \frac{\partial \bar{T}}{\partial \vec{n}}\right|_{C D D_{*} C_{*}}=0, \\
\left.\frac{\partial \bar{T}}{\partial \vec{n}}\right|_{A D D_{*} A_{*} \cup B C C_{*} B_{*} \cup A B C D \cup A_{*} B_{*} C_{*} D_{*}}=0,
\end{gathered}
$$

where $c$ is the concentration of pollution $(\mathrm{ppm}), \bar{T}$ is temperature $\left({ }^{\circ} K\right), D_{\bar{T}}$ is the diffusion coefficient $\left(\mathrm{m}^{2} / \mathrm{s}\right), q_{T}$ is the intensity of internal heat sources, $v_{C}$ is the rate of spread of pollution $(\mathrm{m} / \mathrm{s}), v_{\bar{T}}$ is the rate of spread of temperature $(\mathrm{m} / \mathrm{s})$. The amount of heat $\Psi=\frac{q_{T}}{c_{T} \rho}$ thus released is proportional to the strength of the current, the time of its passage and the voltage drop $q_{T}=I \cdot U \cdot t, U$ is applied voltage $(B), I$ is a current strength $(A), c_{T}$ is a heat capacity $(\mathrm{J} / \mathrm{kg})$, $\rho$ is a density $\left(\mathrm{kg} / \mathrm{m}^{3}\right), 0<x<L$ is a coagulator length $(\mathrm{m}), 0$ $<y<H$ - is a coagulator height $(m), O<z<B$ is a coagulator width $(m)$. During electrocoagulation, the electrolyte solution is heated. The diffusion motion of particles in a fluid can be considered as friction motion, to which the second Einstein relation can be applied: $D_{C}(T)=U_{C} k T$. Here $k$ is Boltzmann constant $(J / K), U_{C}$ mobility of the diffusing particles, that is, the coefficient of proportionality between the velocity of the particle and the driving force $(\mathrm{Pa} \cdot \mathrm{s} \cdot \mathrm{m})$. If the particles are spherically symmetric, then $U_{C}=1 /(6 \pi h r)$ where $h$ is the coefficient of viscosity of the liquid, $r$ is the radius of the particle [3], $\Phi=\frac{I t}{V_{A} n F}+k_{h} S_{k} t$ is the function responsible for the electrochemical reaction, $V_{A}$ here is the volume of the apparatus $\left(M^{3}\right), \mathrm{n}$ is the number of reaction electrons, $F$ is the Faraday constant $(\mathrm{C} / \mathrm{mol}), k_{h}$ is the rate constant of the heterogeneous reaction $\left(\mathrm{mol} / \mathrm{m}^{5} \cdot \mathrm{s}\right), S_{k}$ is the cathode area $\left(\mathrm{m}^{2}\right)$.

\section{EXPERIMENT}

The calculation of the change in the concentration of chromium over time at the exit of the electrocoagulation unit was performed to check the adequacy of the generalized model [5]. The following input data were specified [5]: $L=0.3, m ; \quad B=0.3, m ; \quad H=1, m ; \quad \varepsilon=0.00005 ;$ $D_{c}=0.01, \mathrm{~m}^{2} / \mathrm{min} ; \quad D_{t}=1, \mathrm{~m}^{2} / \mathrm{min} ; \quad V_{1}=0.4 / 60, \mathrm{~m} / \mathrm{min} ;$ $V_{2}=0.4 / 60, \min ; \quad n=2 ; \quad R g=8.314 \cdot 60 \cdot 60, \mathrm{~kg} \cdot \mathrm{m}^{2} / \mathrm{s}^{2}$; $\mu=0.85 ; \quad F=96485.3365, \mathrm{~kg} \cdot \mathrm{m}^{2} / \mathrm{s}^{2} ; d_{b}=1.5 / 1000000, \mathrm{~m} ;$ As $=0.3 \cdot 1 \cdot 10, \mathrm{~m}^{2} ; \quad P=101325 \cdot 60 \cdot 6, \mathrm{~kg} / \mathrm{m} \cdot \mathrm{s}^{2}$; $V_{a}=0.3 \cdot 1 \cdot 0.3, m^{3} ; \quad k_{h}=2.907 ; \quad S_{k}=0.3 \cdot 1 \cdot 10, m^{2}$;
$U=2.71, B ; \quad c_{t}=4218 \cdot 60 \cdot 60,\left(\mathrm{~kg} \cdot \mathrm{m}^{2} / \mathrm{s}^{2}\right) /(\mathrm{kg} \cdot K) ;$ $\rho=1000, \mathrm{~kg} / \mathrm{m}^{3} ; j_{s t}=50, \mathrm{~A} / \mathrm{m}^{2}$.

The peculiarity of this experiment is the size of the reactor $L=0.3, m ; \quad B=0.3, m ; \quad H=1, m ; \quad$ current density $j_{s t 1}=50, A / M^{2}, j_{s t 2}=100, A / M^{2}$. The results of the full-scale experiment and the calculation of the model problem (1) - (2) are presented in Figure 1 and Figure 2.

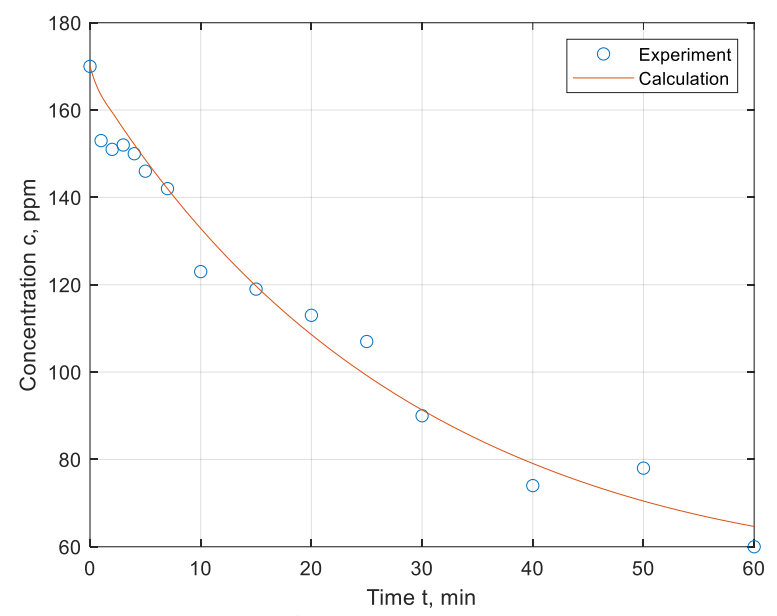

Figure 1. The distribution of chromium concentration over time at the output of the electrocoagulation unit according to the model problem and experiment at a current density of $50 \mathrm{~A} / \mathrm{m}^{2}$ (error $\left.=4.3 \%\right)$.



Figure 2. The distribution of chromium concentration over time at the output of the electrocoagulation unit according to the model problem and experiment at a current density of $100 \mathrm{~A} / \mathrm{m}^{2}($ error $=6.1 \%)$.

Obviously, the electrocoagulation technology for cleaning water from chromium can use several parameters to control the quality of treated water. These parameters according to the proposed model include the speed of fluid flow, the time of the electrocoagulation process, the size of the reactor, the amount of current between the plates, the number of cathodes and anodes, the distance between the plates. There are known researches [4-5] where the influence of the time of the electrocoagulation process, the distance between the plates, the thickness of the cathode and the anode, the current density on the efficiency of chromium extraction from wastewater are 
determined. That is why the investigation of the effect of geometric dimensions of the reactor at a constant volume of the electrocoagulation unit on the extraction of heavy metal ions in the example of chromium is a relevant and urgent task. The main problem that needs to be solved is to make the decision at the design stage in particular about the shape of the electrocoagulation unit, the number of plates, the design dimensions. The minimization of energy consumption during the electrocoagulation process also is an important factor. To study the effect of reactor sizes on the initial concentration of chromium, a calculation was made for different current densities at variable reactor sizes.

The following data were used for the experiment: the sizes of the electrocoagulation $L=0.3, m, B=0.6, m, H=1, m$, as well as all possible combinations, given that in each case the length of the electrocoagulation unit requires a different number of plates with a constant distance between them - 3 $\mathrm{cm}$, current densities, $j_{s t 1}=50, \mathrm{~A} / \mathrm{m}^{2}, j_{s t 2}=100, \mathrm{~A} / \mathrm{m}^{2}$. The simulation results are shown in Figure 3 and Figure 4, and the main characteristics of the process are presented in Table 1.

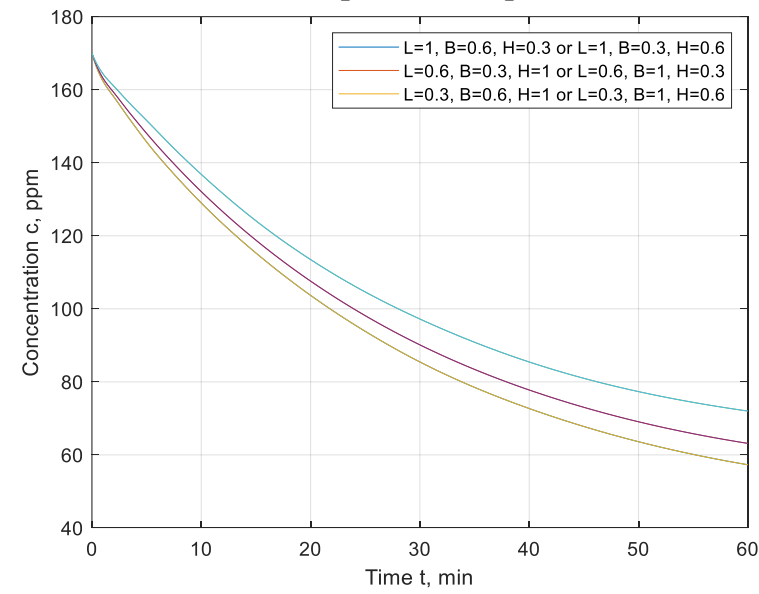

Figure 3. Distribution of chromium concentration over time at the output of the electrocoagulation unit at current density for various design data

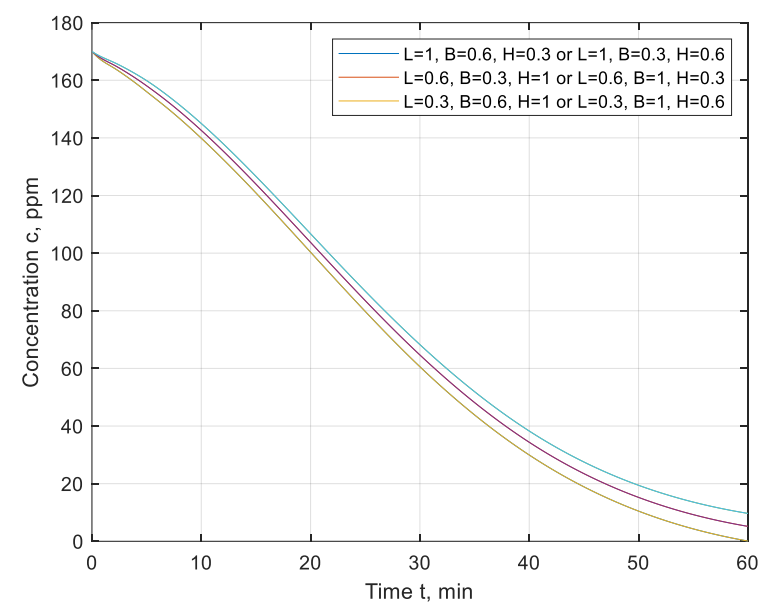

Figure 4. Distribution of chromium concentration over time at the output of the electrocoagulation unit at current density for various design data
Table 1. Variation of chromium concentration at variable current density and size of electrocoagulation unit

\begin{tabular}{|c|c|c|c|}
\hline & \multicolumn{2}{|c|}{$\begin{array}{l}\text { The concentration (ppm) at the output } \\
\text { at time } t=60 \mathrm{~min} \text { at current density: }\end{array}$} \\
\hline & & $50 \mathrm{~A} / \mathrm{m}^{2}$ & $100 \mathrm{~A} / \mathrm{m}^{2}$ \\
\hline \multirow{6}{*}{$\begin{array}{c}\text { Dimensions } \\
\text { of electro- } \\
\text { coagulation } \\
\text { unit }(m)\end{array}$} & $L=1, B=0.3, H=0.6$ & 72.0008 & 9.6835 \\
\hline & $L=1, B=0.6, H=0.3$ & 72.0008 & 9.6835 \\
\hline & $L=0.6, B=1, H=0.3$ & 63.1508 & 5.1835 \\
\hline & $L=0.3, B=1, H=0.6$ & 57.3008 & 0.0835 \\
\hline & $L=0.6, B=0.3, H=1$ & 63.1508 & 5.1835 \\
\hline & $L=0.3, B=0.6, H=1$ & 57.3008 & 0.0835 \\
\hline
\end{tabular}

\section{RESULTS AND DISCUSSION}

To automate the process of electrocoagulation water purification, a functional automation scheme has been developed (see Figure 5), according to which control of the system occurs automatically in real-time mode by the controller using the program algorithm and in the manual mode with operational control exercised by a person. However, regardless of the mode, data are collected on the basic control parameters for archiving, and alarming occurs when exceeding acceptable values, if there are problems in the system.

All functions of regulation and control of basic parameters in the projected automation system are performed by the programmable logic controller S7-300 of Siemens enterprise with modules of expansion, analog inputs SM331, analog outputs SM332 and discrete inputs / outputs SM323, which in this configuration has 2 analog inputs, 2 analog outputs, 8 discrete inputs and 8 discrete outputs that are sufficient to control the system.

To ensure acceptable concentration of chromium in process waters, a system consisting of an electrocoagulation unit, sandbox, primary sump, primary clarifier, aeration pool, 3 sludge level sensors, nickel ion concentration sensor in water, programmable logic controller S7-300, 3 regulators is used, a frequency controlled compressor.

This system has 4 control circuits that provide the following technological parameters at the appropriate level:

1. The concentration of chromium at the outlet of the electrocoagulation unit. (1-analog input, 1-analog output).

2. The level of heavy mineral impurities (sand) in the sand. (1 - discrete input, 1 - discrete output).

3. The level of sediment in the sump. (1 - discrete input, 1 discrete output).

4. The level of sediment in the electrocoagulation unit. (1 discrete input, 1 - discrete output).

Also control the following technological parameters:

1. Control of the rotation of the aeration basin mixer (1 discrete inlet, 1 discrete outlet). 
2. Emergency water level in sandblasts (1 - discrete inlet, 1 - discrete outlet).

3. Emergency water level in the aerator (1 - discrete inlet, 1 - discrete outlet).

4. Emergency water level in sedimentation tanks (1 discrete inlet, 1 - discrete outlet).

In order to detect deviations in the technological process and in case of deviation of critical parameters, to notify the operator for his intervention by turning on the siren. In view of the above, a schematic diagram of the control of chromium concentration in wastewater was developed, which is presented in Figure 5.

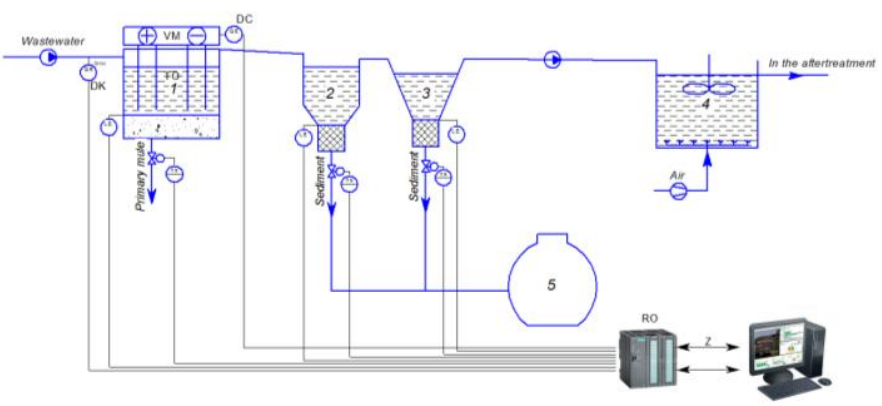

Figure 5. Schematic diagram of control of permissible concentration of impurities in wastewater: 1 - electrocoagulation unit; 2 - primary sump; 3 secondary sump; 4 - aeration pool; 5 - meta tank; $\mathrm{Z}$ - the problem of the permissible concentration of the component to be purified; P1 - standard PI regulator for calculating the current flowing in the electrocoagulation unit; P2

- system PID - regulator for maintaining the set current strength at the appropriate level when compared to the real current flowing between the walls of the electrocoagulation unit; $\mathrm{RO}$ is a regulator that acts as a controller in this system, controlling the magnitude of the applied voltage from the rectifier to the cathode and the anode; VM is an actuator (rectifier) that supplies voltage to the plates of an electrocoagulation unit; TO is a technological object that the reactor stands for; DC is a current encoder that measures the real current

flowing between the walls of an electro coagulation unit; DK is the concentration sensor of the target component at the inlet of the reactor, which shows the change in input concentration for the implementation of the optimal control mode.

The hardware implementation of this project involves the use of a programmable logic controller S-7 300 from Siemens with expansion modules. Using the Simatic Step7 Manager, a process control program was written, preconfiguring the controller hardware and creating a character table that describes all the inputs, outputs, labels with their character, hardware names, and comments. The main components of the program are the implementation of the method of definition and maintenance of the required current between the plates of the electrocoagulation unit. To accomplish this task, we use a standard PID controller, which performs the function of adjusting the current according to the task.

To visualize the project, a graphical screen was created on which automatic and manual control of the process is carried out, display of errors in the operation of the respective devices, as well as display of the main informative parameters of the technological process, such as the direction of the flow of liquid by the glowing green indicator of the respective parts of the valves, filter pumps, also providing information on the applied voltage, current, concentration of chromium at the inlet of the electrocoagulation unit.

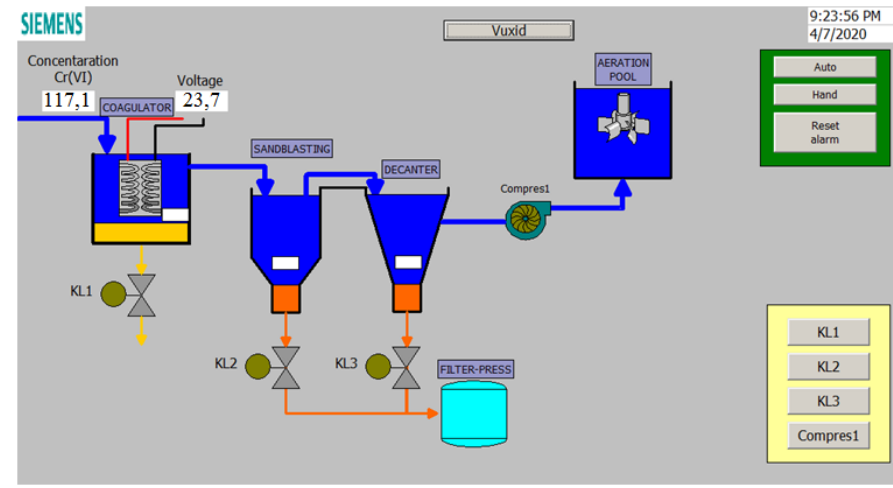

Figure 6. Graphic window for displaying the process of chromium sewage treatment by electrocoagulation

\section{V.CONCLUSION}

A model of the chromium water purification process, which depends on the applied current and the size of the electrocoagulation reactor was developed. The model problem of the corresponding process was solved. The results of calculations of the distribution of chromium concentration over time at the outlet of the coagulator taking into account the change in current and size of the electrocoagulation reactor are presented. The reliability of the model and the obtained results are confirmed by comparison with the results of the visual experiment, and the relative error does not exceed $6.1 \%$. A system of automation of wastewater treatment from chromium, which provides control of the concentration of pollution in the treated wastewater in accordance with the set values was developed. The mnemonic scheme for simplification and efficiency of process management by means of the Simatic - WinCC software package was developed and the set of hardware of automation that will provide reliable and effective work with a wide range of applications was selected.

\section{REFERENCES}

[1] A. P. Safonyk, A. Ya. Bomba, I.M. Tarhonii. Modeling and automation of the electrocoagulation process in water treatment. Advances in Intelligent Systems and Computing, vol 871, 2019, pp 451-463.

[2] A. Safonyk, I. Targoniy, Yu. Martyniuk, A. Bomba. Research and automation of the process of wastewater treatment electrocoagulation. Computer Science and Information Technologies: Proceedings of the XIII International Scientific and technical Conference CSIT 2018.

[3] A. Safonyk, O. Prysiazhniuk. Modeling and simulation in engineering modeling of the electrocoagulation processes in nonisothermal conditions. Modelling and Simulation in Engineering Volume 2019, Article ID 9629643, 2019, p. 6.

[4] A. Safonyk, A. Burduk, I. Targoniy: Research of simulation model of electrocoagulation process. Modeling, Control and Information Technologies (3), 2019, pp. 117-118.

[5] A. Bomba, A. Safonyk, V. Voloshchuk: Spatial modeling of multicomponent pollution removal for liquid treatment under identification of mass transfer coefficient. Mathematical Modeling and Computing Vol. 5, No. 2, 2018, pp. 108-118. 